\title{
Helicopter Vibratory Loads Alleviation through Combined Action of Trailing-Edge Flap and Variable-Stiffness Devices
}

\author{
Massimo Gennaretti, Giovanni Bernardini, Jacopo Serafini, and Marco Molica Colella \\ Department of Engineering, Roma Tre University, Via della Vasca Navale 79, 00146 Rome, Italy \\ Correspondence should be addressed to Jacopo Serafini; serafini@uniroma3.it
}

Received 12 March 2015; Accepted 21 May 2015

Academic Editor: R. Ganguli

Copyright ( 2015 Massimo Gennaretti et al. This is an open access article distributed under the Creative Commons Attribution License, which permits unrestricted use, distribution, and reproduction in any medium, provided the original work is properly cited.

\begin{abstract}
The aim of this paper is the assessment of the capability of controllers based on the combined actuation of flaps and variable-stiffness devices to alleviate helicopter main rotor vibratory hub loads. Trailing-edge flaps are positioned at the rotor blade tip region, whereas variable-stiffness devices are located at the pitch link and at the blade root. Control laws are derived by an optimal control procedure based on the best trade-off between control effectiveness and control effort, under the constraint of satisfaction of the equations governing rotor blade aeroelastic response. The numerical investigation concerns the analysis of performance and robustness of the control techniques developed, through application to a four-bladed helicopter rotor in level flight. The identification of the most efficient control configuration is also attempted.
\end{abstract}

\section{Introduction}

This work deals with the analysis of control systems devoted to the alleviation of helicopter vibratory hub loads. Indeed, rotational and translational motion combined with the cyclic pitch control and with the resulting complex aerodynamic environment in which helicopter rotor blades operate (wake inflow, dynamic stall, and reverse-flow effects) cause blade periodic deformations and transmission of vibratory loads to the hub and hence to the fuselage. In turn, fuselage vibrations produce acoustic disturbances inside the cabin that may cause discomfort and have a significant impact on the fatigue-life of the structure (and hence on maintenance costs). In addition, these have a negative impact on functionality of instruments located onboard, also making their reading difficult. All this explains why the suppression/alleviation of vibratory hub loads is one of the critical (and most challenging) goals in helicopter design and has received increased attention by rotorcraft researchers in the last decade.

Several approaches to vibratory hub loads reduction have been investigated in detail in the recent past [1-10]. In particular, two types of control systems have been considered: the individual blade control (IBC), in which each blade is controlled separately, and the higher harmonic control
(HHC), which acts simultaneously on all the blades through actuators installed underneath the swashplate. Benefits of these systems in reducing vibrations have been widely analysed, with the IBC approach proven to be potentially more effective than the HHC which, in turn, presents advantages in terms of technological simplicity. In addition, IBC is capable of actuating any multiple frequencies of the revolution one, whereas the HHC provides a limited set of control multiple frequencies in the rotating frame, in that working through actuation of the nonrotating component $[6,11,12]$.

Starting from these considerations, in this work an individual blade control is considered for reducing vibrations. It is based on the combined use of variable-stiffness devices [13] and trailing-edge flaps. In particular, the variable-stiffness devices are assumed to be embedded both at the blade root to modify the bending stiffness and in the pitch link to modify the torsional dynamics of the blade. These devices, named smart springs [13], are based on adaptive material to provide stiffness variation requiring very low actuation power (see $[13,14]$ for details).

A sketch of the blade with inclusion of these control devices is given in Figure 1. Specifically, the investigation is aimed at the assessment of both effectiveness and robustness of such a control technique. The laws of the cyclic stiffness 


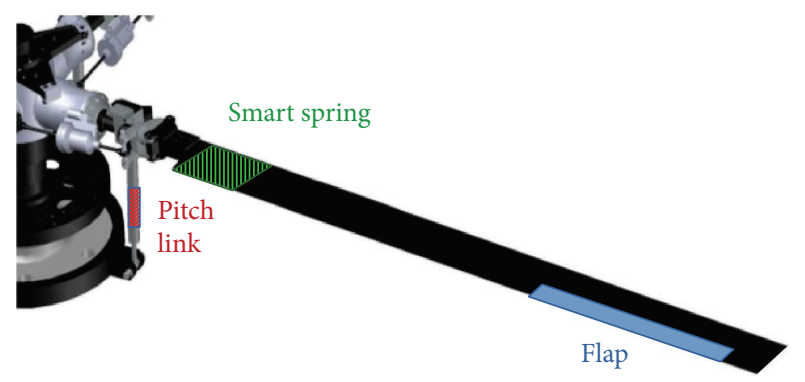

FIgURE 1: Blade with flap and variable-stiffness devices.

variation and flap deflection are obtained by an optimal control based on the minimization of a cost function which includes hub loads and control input harmonics, under the constraint of compatibility with equations governing the blade aeroelasticity.

Blade aeroelastic modeling is derived by coupling structural dynamics equations with unsteady aerodynamic loads. The blade structural dynamics is described through the nonlinear flap-lag-torsion equations of motion presented by Hodges and Dowell [15]. These are related to a beamlike model and are valid for straight, slender, homogeneous, isotropic, nonuniform, and twisted blades undergoing moderate deflections. Akin to several rotor prediction tools commonly used by helicopter industries, the aerodynamic loads are simulated through quasi-steady, sectional aerodynamic models including flap effects, with downwash wake inflow corrections obtained either analytically or numerically evaluated by a three-dimensional, free-wake, Boundary Element Method (BEM) tool for unsteady potential flows [16]. The final aeroelastic equations are solved through the Galerkin method, followed by a harmonic balance approach to the evaluation of periodic responses [17]. Vibratory hub loads are evaluated by integration of inertial and aerodynamic loads distributed along each blade span.

The numerical investigation examines effectiveness and robustness of control techniques based on several combinations of trailing-edge flap and variable-stiffness devices, considering the vibratory hub loads arising in a four-bladed rotor in level forward flight. The study will attempt to identify the most efficient control strategy.

\section{Blade Structural Dynamics Model}

The starting point for the identification of optimal control actuation of blade flap and variable-stiffness devices is the definition of the aeroelastic model of the helicopter rotor: it is essential both in the control-law synthesis process and for the validation of the performance of the controller.

The blade structural dynamics equations are based on the nonlinear bending-torsion formulation presented by Hodges and Dowell [15], which is valid for straight, slender, homogeneous, isotropic, nonuniform, and twisted blades, undergoing moderate displacements. Indeed, second-order terms are retained in the equations after the application of an ordering scheme that drops third-order terms (with respect to bending slope) not contributing to damping. For a rotating hub-centered orthogonal coordinate system having the $x$ axis aligned with the undeformed elastic axis and the $y$-axis parallel to the plane of rotation, the blade dynamics is governed by the following integropartial differential equations in terms of elastic axis displacements and cross section torsion (Figure 2):

$$
\begin{aligned}
& {\left[\left(\Lambda_{2}-\Lambda_{21} \sin ^{2} \theta\right) v^{\prime \prime}\right]^{\prime \prime}} \\
& +\Lambda_{21}\left[\phi w^{\prime \prime} \cos (2 \theta)-\phi v^{\prime \prime} \sin (2 \theta)\right]^{\prime \prime} \\
& +\frac{\Lambda_{21}}{2}\left[w^{\prime \prime} \sin (2 \theta)\right]^{\prime \prime}-\left(T v^{\prime}\right)^{\prime} \\
& -e_{A} T \cos (\theta+\phi)=L_{v}(v, w, \phi, \beta)+p_{y}(v, w, \phi, \beta) \\
& -q_{z}^{\prime}(v, w, \phi, \beta) \\
& {\left[\left(\Lambda_{1}+\Lambda_{21} \sin ^{2} \theta\right) w^{\prime \prime}\right]^{\prime \prime}} \\
& +\Lambda_{21}\left[\phi v^{\prime \prime} \cos (2 \theta)+\phi w^{\prime \prime} \sin (2 \theta)\right]^{\prime \prime} \\
& +\frac{\Lambda_{21}}{2}\left[v^{\prime \prime} \sin (2 \theta)\right]^{\prime \prime}-\left(T w^{\prime}\right)^{\prime} \\
& -e_{A} T \sin (\theta+\phi)=L_{w}(v, w, \phi, \beta)+p_{z}(v, w, \phi, \beta) \\
& +q_{y}^{\prime}(v, w, \phi, \beta) \text {, } \\
& \Lambda_{21}\left[\left(w^{\prime \prime 2}-v^{\prime \prime 2}\right) \sin \theta \cos \theta+v^{\prime \prime} w^{\prime \prime} \cos (2 \theta)\right]-\kappa \phi^{\prime \prime} \\
& -\left[\eta^{2} K T(\phi+\theta)^{\prime}\right]^{\prime}-e_{A} T\left(w^{\prime \prime} \cos \theta-v^{\prime \prime} \sin \theta\right) \\
& +\kappa_{\mathrm{pl}} \phi \delta\left(x-x_{h}\right)=M_{\phi}(v, w, \phi, \beta)+q_{x}(v, w, \phi, \beta) \\
& +v^{\prime} q_{y}(v, w, \phi, \beta)+w^{\prime} q_{z}(v, w, \phi, \beta),
\end{aligned}
$$

where the tension, $T$, is given by

$$
T=\int_{x}^{1} p_{x}(v, w, \phi) d \bar{x}
$$

and ()$^{\prime}$ denotes derivation with respect to dimensionless spanwise coordinate. The spanwise distributions of inertial forces, $p_{x}, p_{y}, p_{z}$, inertial moments, $q_{x}, q_{y}, q_{z}$, and aerodynamic loads, $L_{v}, L_{w}, M_{\phi}$, are functions of the blade section degrees of freedom. In the equations above, dimensionless lengths are related to the blade radius, $R$, while dimensionless time coincides with blade azimuth position. The bending equations (1) and (2) have been obtained by division by the parameter $m \Omega^{2} R$, while the torsion equation (3) is the result of division by the parameter $m \Omega^{2} R^{2}$, with $m$ and $\Omega$ denoting the reference blade mass per unit length and the blade angular velocity, respectively. Moreover, $\Lambda_{1}$ and $\Lambda_{2}$ are the dimensionless flap and lead-lag bending stiffness, $\Lambda_{21}=\Lambda_{2}-\Lambda_{1}, \kappa$ is the dimensionless torsion rigidity, $\eta$ is the dimensionless sectional mass radius of gyration, and $K$ is the square of the ratio between the blade cross section polar radius of gyration and the blade cross section mass radius of 


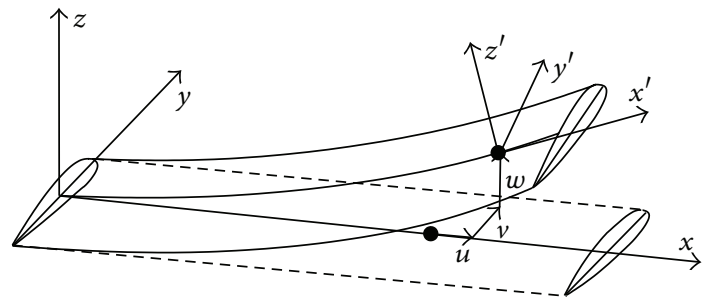

(a) Blade isometric view

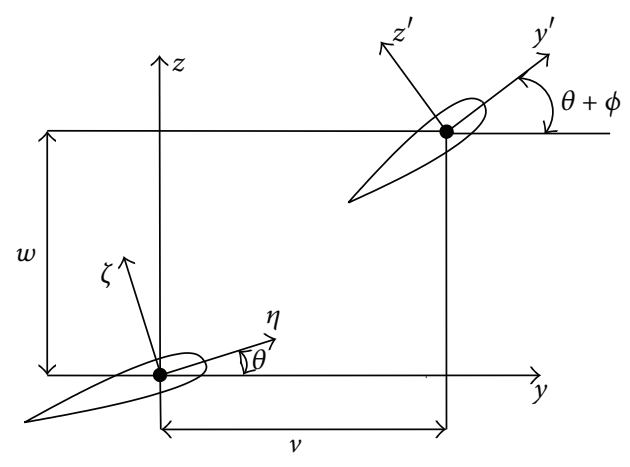

(b) Blade section

FIGURE 2: Rotor blade coordinate systems and displacements.

TABLE 1: Nondimensionalization factors.

\begin{tabular}{lc}
\hline Physical quantities & Scaling factor \\
\hline Length & $R$ \\
Stiffness & $m \Omega^{2} R^{4}$ \\
Force per unit length & $m \Omega^{2} R$ \\
Moment per unit length & $m \Omega^{2} R^{2}$ \\
Generalized force & $m \Omega^{2} R^{3}$ \\
\hline
\end{tabular}

gyration, whereas $\theta$ is the section pitch angle and $e_{A}$ is the tensile offset (see [15] for details and Table 1 where scaling factors are summarized).

The effects of the control actuation of trailing-edge flap and variable-stiffness devices located at blades root and pitch link are included in (1)-(3). In particular, the angle $\beta$ denotes flap deflection which affects aerodynamic and inertial loads at those blade sections where it is present. The influence of the pitch link variable-stiffness device is simulated as a time-varying torsional spring, $\kappa_{\mathrm{pl}}$, at the pitch hinge location, $x_{h}$ (see (3)). Embedded smart springs devoted to change bending stiffness are located in the blade root region, $x_{i}<x<$ $x_{e}$, and their effects are simulated assuming that $\Lambda_{1}=\Lambda_{1}^{0}+$ $\Delta \Lambda_{1}\left[H\left(x-x_{i}\right)-H\left(x-x_{e}\right)\right]$ and $\Lambda_{2}=\Lambda_{2}^{0}+\Delta \Lambda_{2}\left[H\left(x-x_{i}\right)-\right.$ $H\left(x-x_{e}\right)$ ], with $\Lambda_{1}^{0}$ and $\Lambda_{2}^{0}$ denoting the baseline bending stiffness, $\Delta \Lambda_{1}$ and $\Delta \Lambda_{2}$ their spanwise uniform variations, and $H$ representing the Heaviside step function.

\section{Blade Aerodynamics Model}

As stated above, here the aerodynamic loads on the blade are analyzed by using a quasi-steady sectional model, with downwash affected by wake inflow (mainly, to take into account three-dimensional effects due to the trailed vortices released by the rotor blades). It is based on the Theodorsen [18] and Greenberg [19] theories, under the assumption of very low frequency analysis, for which the lift deficiency function is constant and equal to one.

Specifically, denoting by $u_{T}$ and $u_{P}$ the components of the elastic axis velocity along the section principal axes $\left(u_{T}\right.$ is chordwise and $u_{P}$ is normal to chord, upward) and by $\omega$ the section angular velocity orthogonal to the section plane, distributed lift is given by $[18,19]$

$$
L=L_{n c}+L_{c},
$$

where noncirculatory lift, $L_{n c}$, and circulatory lift, $L_{c}$, are, respectively,

$$
\begin{aligned}
L_{n c} & =\rho b^{2}\left[\pi\left(\frac{\dot{\omega} b}{2}-\dot{u}_{P}\right)-T_{4}\left(\dot{u}_{T} \beta+u_{T} \dot{\beta}\right)-b T_{1} \ddot{\beta}\right], \\
L_{c} & =2 \rho b^{2} u_{T} C(k)\left[\pi\left(\omega-\frac{u_{P}}{b}\right)+\frac{T_{10}}{b} u_{T} \beta+\frac{T_{11}}{2} \dot{\beta}\right]
\end{aligned}
$$

while distributed aerodynamic moment about the $1 / 4$-chord point reads $[18,19]$

$$
\begin{aligned}
M_{\phi} & =-\rho b^{3}\left\{\frac{\pi}{2}\left(-\dot{u}_{P}+u_{T} \omega+\frac{3}{4} b \dot{\omega}\right)\right. \\
& +\left[\frac{\left(T_{4}+T_{10}\right) u_{T}^{2}}{b}-T_{8} \dot{u}_{T}-\left(\bar{c}+\frac{1}{2}\right) T_{4} \dot{u}_{T}\right] \beta \\
& +u_{T}\left[T_{1}-T_{8}-\left(\bar{c}+\frac{1}{2}\right) T_{4}+\frac{1}{2} T_{11}\right] \dot{\beta} \\
& \left.-b\left[T_{7}+\left(\bar{c}+\frac{1}{2}\right) T_{1}\right] \ddot{\beta}\right\} .
\end{aligned}
$$

In the equations above, $\rho$ is the air density, $b$ is the blade section semichord length, $\bar{c}$ is the trailing-edge flap chord, and $C(k)$ is the lift deficiency function with $k$ denoting the reduced frequency, while the $T_{j}^{\prime}$ s are Theodorsen's coefficients (see [18] for details). Next, introduce the drag section force:

$$
D=\rho b\left(u_{T}^{2}+u_{P}^{2}\right) c_{D},
$$

where $c_{D}$ is the profile drag coefficient. Then, for $\alpha=\theta+\phi$, the distributed loads forcing blade lagging and flapping read (see, e.g., [20])

$$
\begin{aligned}
& L_{v}=-L\left(\frac{u_{P}}{u_{T}}+\alpha\right)-D, \\
& L_{w}=L-D\left(\frac{u_{P}}{u_{T}}+\alpha\right) .
\end{aligned}
$$


Note that the velocity components, $u_{T}$ and $u_{P}$, are the result of the combination of rigid blade motion and elastic deformation (and thus are functions of $v, w, \phi$ ) but also include the effects of the inflow velocity induced by the rotor wake. Here, the wake inflow on the rotor disk is obtained either analytically [21] or numerically by a three-dimensional, free-wake, BEM computational tool for unsteady potential flows [16].

\section{Blade Aeroelastic Model}

Combining (1)-(3) with the aerodynamic loads yields the rotor aeroelastic model to be solved. The space integration of the equations is performed through the Galerkin method, based on the description of the elastic deformation as a linear combination of suitable linearly independent shape functions that satisfy the homogeneous boundary conditions corresponding to the structural constraints (e.g., for a hingeless blade they are chosen as bending natural modes of a cantilever beam). The resulting aeroelastic system consists of a set of nonlinear ordinary differential equations of the type

$$
\mathbf{M}(t) \ddot{\mathbf{q}}+\mathbf{C}(t) \dot{\mathbf{q}}+\mathbf{K}(t) \mathbf{q}=\mathbf{f}_{\mathrm{str}}^{\mathrm{nl}}(t, \mathbf{q})+\mathbf{f}_{\mathrm{aer}}(t, \mathbf{q}),
$$

where $\mathbf{q}$ denotes the vector of the Lagrangean coordinates of blade, whereas $\mathbf{M}, \mathbf{C}$, and $\mathbf{K}$ are time-periodic, mass, damping, and stiffness structural matrices representing the linear structural terms. Nonlinear structural contributions are collected in $\mathbf{f}_{\text {str }}^{\mathrm{nl}}$, whereas the generalized aerodynamic forces are collected in $\mathbf{f}_{\mathrm{aer}}$. Note that the control variables, $\beta, \Delta \Lambda_{1}, \Delta \Lambda_{2}$, and $\kappa_{\mathrm{pl}}$, mainly affect generalized aerodynamic forces and stiffness matrix. Finally, the aeroelastic responses are determined through a harmonic balance approach. It consists of the following: (i) expressing LHS and RHS of (10) in terms of Fourier series; (ii) equating the resulting coefficients; and (iii) solving the corresponding algebraic set of equations in terms of the unknown Fourier coefficients of the Lagrangean coordinates of the problem. Note that, because of the presence of nonlinear terms, the harmonic balance solution requires an iterative procedure [17].

Once the steady-periodic blade deformations are determined, the hub loads are evaluated by integrating inertial and aerodynamic loads along the blade span and combining the contributions from each blade.

\section{Identification of Optimal Control Law}

Following an approach already used in the past by other authors that have faced the problem of helicopter vibration control [1,22-24], different optimal control laws are identified in this paper, minimizing the following performance index:

$$
J=\mathbf{z}^{T} \mathbf{W}_{z} \mathbf{z}+\mathbf{u}^{T} \mathbf{W}_{u} \mathbf{u},
$$

where $\mathbf{u}$ is the vector collecting the control input amplitudes to be determined (combination of harmonics of flap deflection, pitch link, and blade root bending stiffness, in our case) and $\mathbf{z}$ is the vector of the quantities to be reduced (hub loads harmonics, in this problem), while $\mathbf{W}_{z}$ and $\mathbf{W}_{u}$ are weighting matrices that are defined so as to get the best trade-off between control effectiveness and control effort. Because of the inherently time-periodic nature of the problem, this control approach involves only the harmonics of input and output variables, without concerning the evolution of transients. In the present problem, for an $N$-bladed rotor, the output vector consists of the $N /$ rev. sine and cosine harmonics of forces and moments at the hub, while the control inputs are the sine and cosine harmonics of (i) flap deflection, $\beta$, (ii) pitch link stiffness, $\kappa_{\mathrm{pl}}$, and (iii) blade root smart spring stiffness variables, $\Delta \Lambda_{1}$ and $\Delta \Lambda_{2}$, that are effective for control.

Akin to the standard optimal LQR control method (of which the present approach may be interpreted as the natural extension for the application to the control of the steady-periodic behavior of a system governed by nonlinear, periodic-coefficient differential equations), the minimization of the cost function is obtained under the constraint of satisfying the governing equation of the system controlled. Such constraint is not directly represented by (10) but rather is given by the following linearized relationship (about a reference input state, $\mathbf{u}_{n-1}$ ) between control inputs, $\mathbf{u}$, and system response, $\mathbf{z}$ :

$$
\mathbf{z}_{n}=\mathbf{z}_{n-1}+\mathbf{T}_{n-1}\left(\mathbf{u}_{n}-\mathbf{u}_{n-1}\right),
$$

where $\mathbf{T}_{n-1}$ is the (Jacobian) transfer matrix that may be obtained numerically from solutions of (10). Note that the nonlinear behavior of the rotor aeroelastic response implies that the transfer matrix is not constant but, rather, is a function of the reference input state, $\mathbf{u}_{n-1}$. Then, combining (11) with (12) and minimizing the resulting cost function yield the following optimal control input:

$$
\mathbf{u}_{n}=\mathbf{G}_{u} \mathbf{u}_{n-1}-\mathbf{G}_{z} \mathbf{z}_{n-1},
$$

where the gain matrices are given by

$$
\begin{aligned}
\mathbf{G}_{u} & =\mathbf{D T}_{n-1}^{T} \mathbf{W}_{z} \mathbf{T}_{n-1}, \\
\mathbf{G}_{z} & =\mathbf{D T}_{n-1}^{T} \mathbf{W}_{z}
\end{aligned}
$$

with

$$
\mathbf{D}=\left(\mathbf{T}_{n-1}^{T} \mathbf{W}_{z} \mathbf{T}_{n-1}+\mathbf{W}_{u}\right)^{-1}
$$

Equation (13) has to be used in a recursive way: starting from the uncontrolled output, the gain matrices are implemented in a closed-loop control process in which, at each $n$th control step, measured vibratory hub loads and corresponding control inputs are used as a feedback to update the control law; the time interval between each control step should be long enough to allow the helicopter to reach the steady-periodic state corresponding to the updated controls [22]. The optimal controller law is updated until convergence. Alternatively, for semiactive, open-loop control, the input may be derived by the following (nonrecursive) reduced version of (13):

$$
\mathbf{u}=-\mathbf{G}_{z} \mathbf{z}_{0}
$$

where $\mathbf{G}_{z}$ is obtained by evaluating $\mathbf{T}$ at $\mathbf{u}=0$ (gain scheduling approach). 
In the recent past, this multiharmonic optimal controller has been successfully applied by the authors in problems concerning the alleviation of Blade-Vortex Interaction noise, vibratory hub loads of helicopters and tiltrotors, and interior noise of propeller-driven aircraft and tiltrotors (see, e.g., [2527]).

\section{Numerical Results}

The control approach presented above is applied to alleviate the vibratory hub loads arising during level flight of a Bo-105like four-bladed main rotor. The radius of this rotor is $R=$ $4.93 \mathrm{~m}$, the blades have constant chord, $c=0.395 \mathrm{~m}$, and linear twist angle is equal to $-8^{\circ}$; in addition, bending and torsional nondimensional stiffness (as well as cross section mass radius of gyration and mass per unit length) have been assumed to be uniformly distributed along the blade span and, respectively, equal to $\Lambda_{1}=0.008345, \Lambda_{2}=0.023198$, and $\kappa=0.00225$ (see [23], for further details). A flight configuration with advance ratio $\mu=0.3$ has been examined, with trim pitch controls related to blade rotational speed $\Omega=$ $40 \mathrm{rad} / \mathrm{s}$. The trailing-edge blade flaps actuated in the control procedure have chord $\bar{c}=0.2 c$, span $b_{f}=0.2 R$, with the midsection located at $70 \%$ blade span position.

In the first step of the analysis, the control matrices, $\mathbf{G}_{u}$ and $\mathbf{G}_{z}$, are identified as those yielding the best hub loads reduction, under the limitation of requiring a maximum flap deflection of $\pm 4^{\circ}$, maximum amplitudes of flap and lead-lag bending stiffness cyclic variations equal to $10 \%$ of $\Lambda_{1}$ and $\Lambda_{2}$, respectively, and maximum amplitude of pitch link stiffness variation equal to $10 \%$ of $\kappa_{\mathrm{pl}}$ (such constraints are introduced to take into account the operational limitations that would unavoidably arise during the practical implementation of the controller).

Specifically, considering an aeroelastic rotor model based on the wake inflow given by Drees [21], the optimal weighting matrices and the corresponding control variables cyclic evolution have been determined using a local controller approach, with the transfer matrix in (12) given by a prior analytical approximation based on a least square procedure [10]. Indeed, this approach allows a search of the optimal control law that is almost as fast as that achievable using a global controller (i.e., considering a constant transfer matrix) and much faster than the local controller ones based on the numerical evaluation of the transfer matrix at each step of the control process. Note that the application of a local controller is needed because of the presence of the trailingedge flap deflection among the control variables: indeed, due to the presence of significant nonlinearities, the elements of the transfer matrix related to the flap action are those more dependent on the control variables state about which they are evaluated.

First, in order to validate the controller identified, the control laws are applied in open-loop mode (see Section 5) to alleviate the vibratory hub loads as predicted by the same rotor aeroelastic model used in the controller-synthesis phase. In particular, Figure 3 compares the baseline vibratory hub loads (i.e., without any control actuation) with those

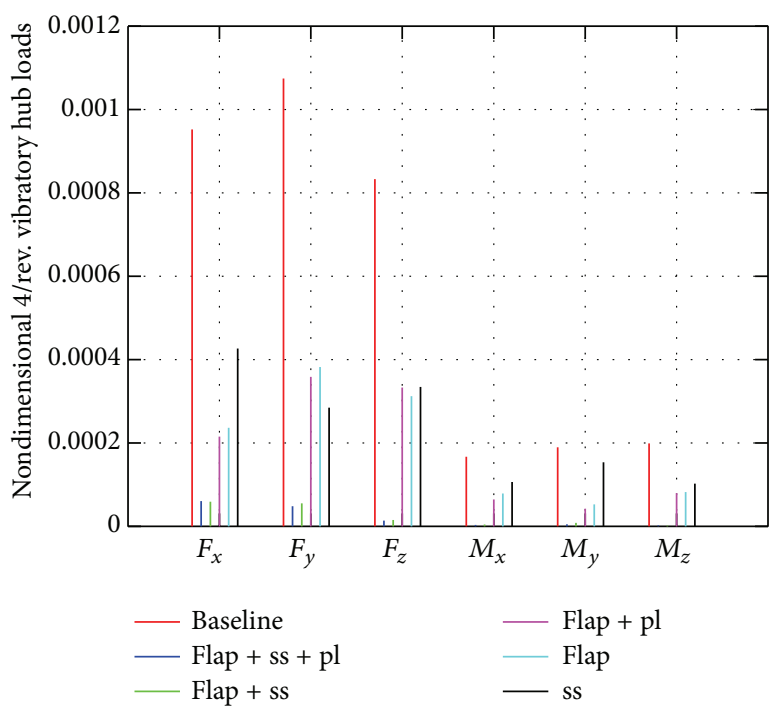

Figure 3: Hub loads, Drees wake inflow.

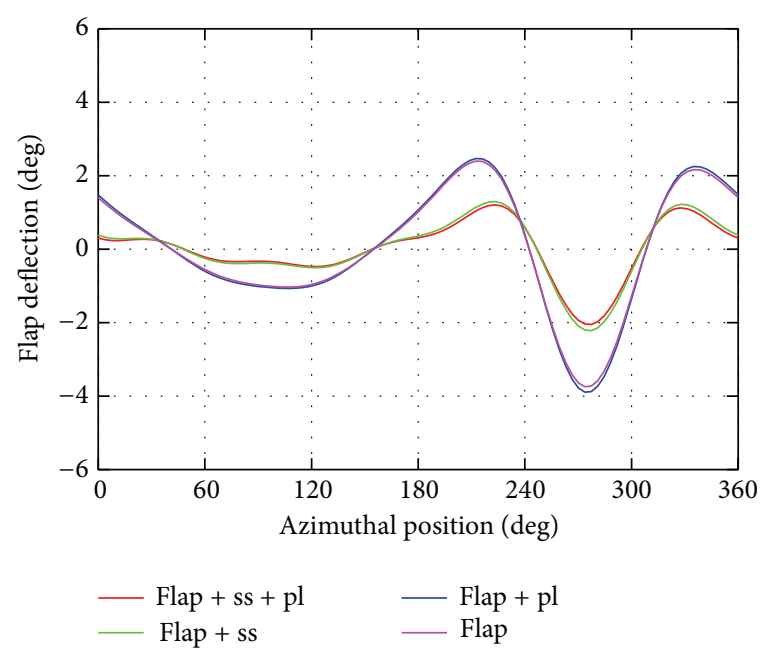

FIGURE 4: Flap deflections time history.

resulting from the application of the controller acting through different combinations of the control variables considered in this work (namely, flap deflections, and stiffness variations of lead-lag and flap smart springs (ss) and pitch link (pl)). Figure 4 depicts the flap deflection time histories used in the control procedures applied for the results in Figure 3, while Figures 5-7 show the corresponding bending stiffness and pitch link stiffness variations during one blade revolution (in this analysis the $2-5 / \mathrm{rev}$. harmonics of the control variables have been considered, in that these are the most effective for a four-bladed rotor control). It is worth noting that Figure 4 shows a sudden upward deflection of the flap around the azimuthal position $\psi=270^{\circ}$. This reduces the high angles of attack occurring in the retreating side, due to both blade kinematics and pitch control, thus tending to provide a more uniform azimuthal distribution of blade loads. Conversely, the pitch link stiffness is considerably 


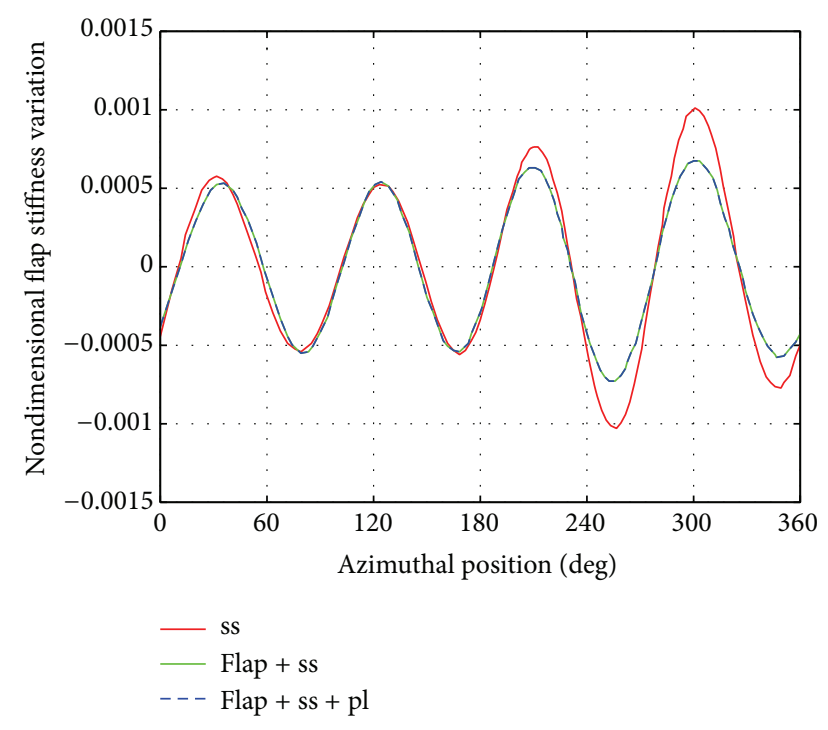

FIgURE 5: Flap bending stiffness time history.

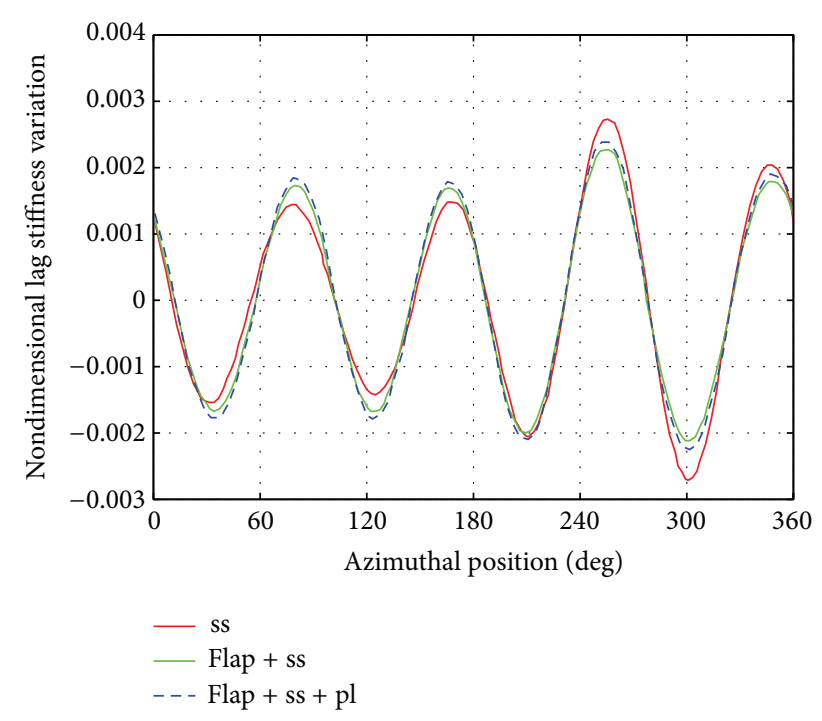

FIGURE 6: Lag bending stiffness time history.

reduced in the advancing side (see Figure 7): this might be due to the phase lag between transfer functions relating flap and pitch link actuation to hub loads, which implies a phase lag between their actuations to pursuit the same vibration reduction goal.

All the control approaches applied seem to yield significant alleviations of the vibratory loads; however, the most effective strategies are those based on the combined use of cyclic trailing-edge flap deflections and blade root stiffness variations through smart springs. In addition, the control laws based on such combinations require lower control variables effort with respect to the other techniques (see Figures 4-7). The results given by the application of the only pitch link stiffness variation have not been included in that, at least for the stiffness range that has been considered in

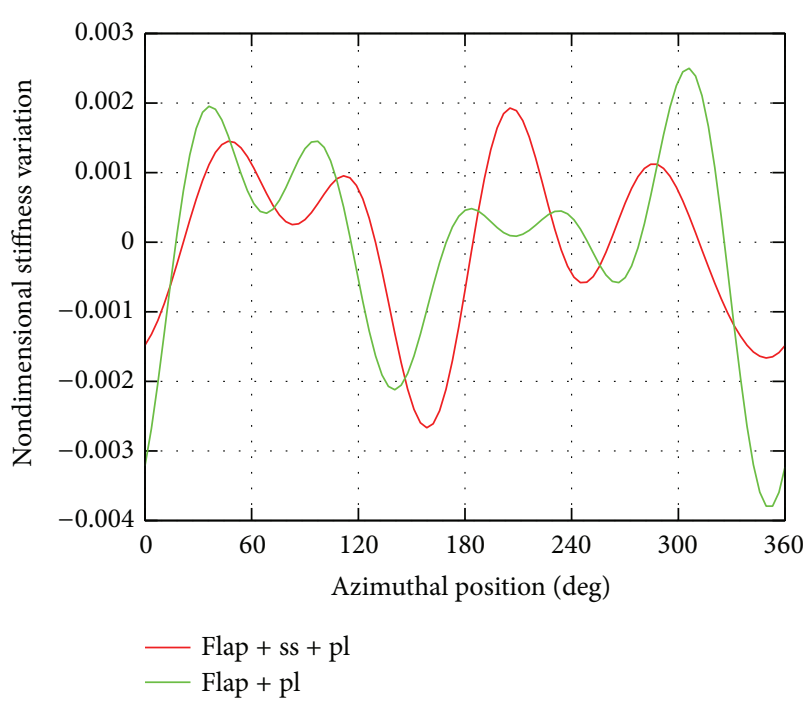

FIgURE 7: Pitch link stiffness time history.

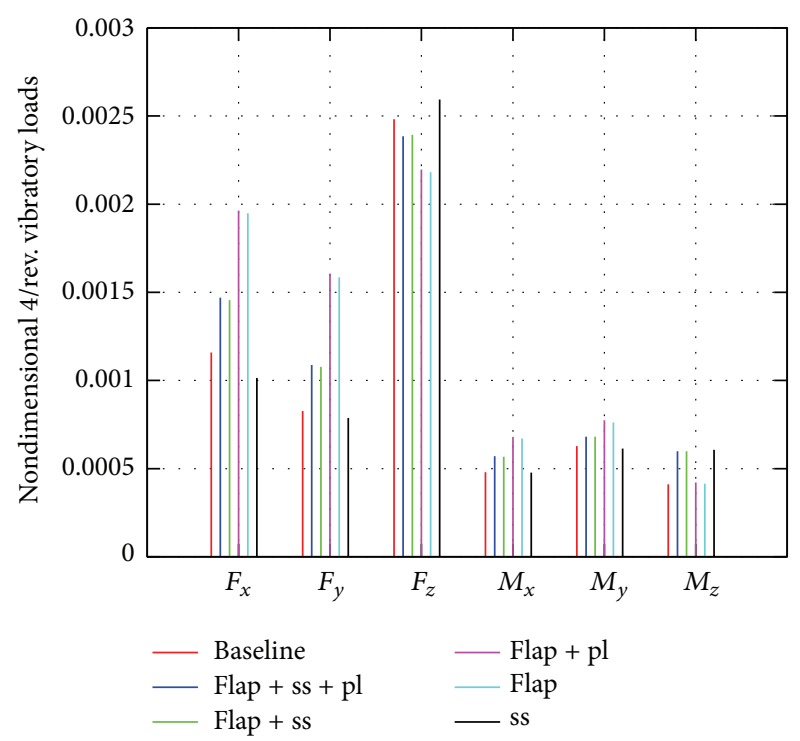

FIgURE 8: Hub loads without feedback, Drees synthesis, and freewake inflow validation.

this work, it does not produce significant vibratory loads reductions.

Then, the robustness of the control laws is analysed by upgrading the aeroelastic model used in the validation phase, with respect to that used in the control synthesis. Specifically, the cyclic control variables histories are applied to a rotor aeroelastic model that uses a more realistic wake inflow based on a BEM free-wake aerodynamic solver [16]. In this case, the effectiveness of the control techniques dramatically changes. This is demonstrated by Figure 8 that shows the inefficiency of the controller which, for some loads, even increases the vibratory level. Indeed, with respect to the model based on the Drees wake inflow, the application of a wake inflow based on a free-wake solver introduces higher frequencies in the aerodynamic loads and hence significantly 
modifies the harmonic loads in the rotating frame and hence the vibratory hub loads (note that the baseline vibratory hub loads in Figure 8 are significantly higher than those in Figure 3). Thus the cyclic controls identified by the simpler aeroelastic model may result as unsuitable. The numerical synthesis of open-loop control laws may provide control strategies producing unsatisfactory performance in the realworld applications. This problem may be alleviated, but not completely eliminated, by improving the accuracy of the numerical tools used in the control synthesis phase. Next, we examine a strategy to overcome this problem that includes a feedback process in the definition of the flap actuation and analyse the benefit of using more accurate simulations in the control identification process. In particular, leaving the actuation laws regarding the smart springs at blade root and pitch link that have been identified by the simpler aeroelastic model unchanged, (13) is applied recursively to determine the flap deflection that minimizes the performance index for the given (feedback) vibratory hub loads (note that if stiffness control variables are also updated in the feedback control process, the procedure does not tend to a converged solution).

Figure 9 shows the vibratory hub loads corresponding to the different control strategies examined here, with the application of the feedback process in the flap actuation law. Percentage variations with respect to the baseline values are summarized in Table 2. In this case the results are quite satisfactory, although it is unclear if it is useful to combine flap with root smart spring, rather than controlling with the only trailing-edge flap. A clearer answer to such question comes from Figure 10 and Table 3. They present the hub loads alleviation using the feedback process for the flap actuation with control matrices $\mathbf{G}_{u}$ and $\mathbf{G}_{z}$ in (13) and the cyclic stiffness variations identified with an aeroelastic simulation based on the wake inflow given by a BEM prescribed (undistorted) wake solver. The improved accuracy of the aeroelastic solver applied in the control-law identification yields a better performance of the control procedures examined. However, in this case, due to the increased reliability of the cyclic stiffness actuation laws, the combined use of flap and blade root smart spring seems to be the most efficient control procedure.

The cyclic flap deflections corresponding to the controlled hub loads in Figure 10 are depicted in Figure 11, with their harmonic content given in Figure 12. Differently from control law based on Drees wake inflow model, here the flap deflection shows a sudden decrease at azimuthal positions around $\psi \approx 180^{\circ}$ and $\psi \approx 360^{\circ}$, for all the combinations of the actuation devices. This difference is probably due to the more realistic wake inflow models considered, which produce a highly nonuniform wake inflow distribution on the rotor disk. However, these results confirm the importance of the $2 / \mathrm{rev}$. flap motion in the $4 / \mathrm{rev}$. vibratory loads alleviation [11], although the high-frequency contributions seem to be not negligible at all.

Figures 13 and 14 depict the cyclic blade root bending stiffness variations that are identified by the prescribed-wake inflow aeroelastic model and correspond to the hub loads in Figure 10. It is worth noting that Figure 13 shows a phase shift of the flap bending stiffness when the smart spring is used in combination with other control devices, whereas

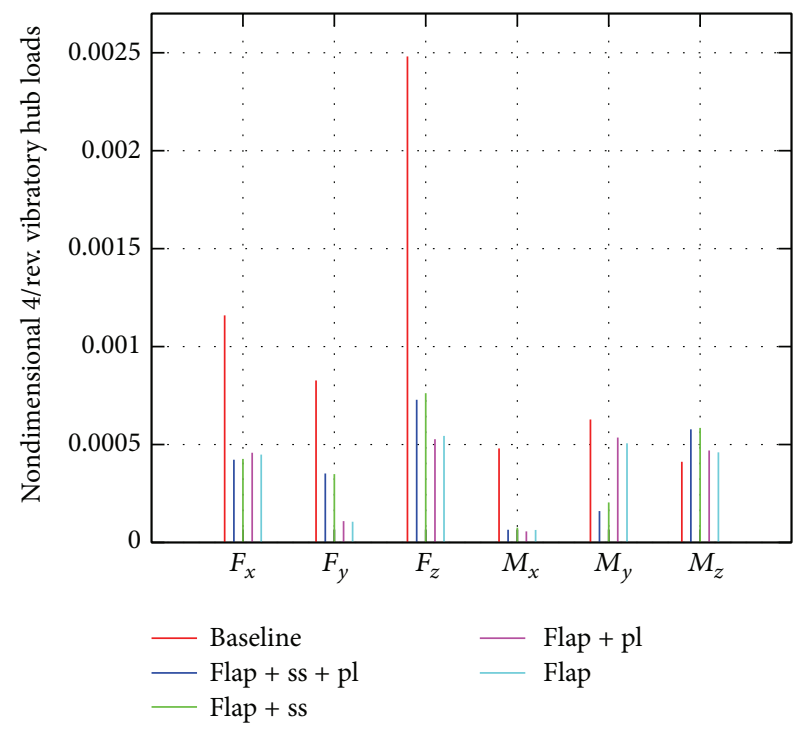

FIGURE 9: Hub loads with feedback, Drees synthesis, and free-wake inflow validation.

TABLE 2: Reductions of hub loads with feedback (Drees synthesis).

\begin{tabular}{lcccccc}
\hline & $F_{x}$ & $F_{y}$ & $F_{z}$ & $M_{x}$ & $M_{y}$ & $M_{z}$ \\
\hline Flap + ss + pl & $-64 \%$ & $-58 \%$ & $-71 \%$ & $-86 \%$ & $-74 \%$ & $+40 \%$ \\
Flap + ss & $-63 \%$ & $-57 \%$ & $-69 \%$ & $-84 \%$ & $-68 \%$ & $+42 \%$ \\
Flap + pl & $-60 \%$ & $-87 \%$ & $-79 \%$ & $-88 \%$ & $-15 \%$ & $+14 \%$ \\
Flap & $-61 \%$ & $-87 \%$ & $-78 \%$ & $-86 \%$ & $-19 \%$ & $+11 \%$ \\
\hline
\end{tabular}

TABLE 3: Reductions of hub loads with feedback (prescribed-wake synthesis).

\begin{tabular}{lcccccc}
\hline & $F_{x}$ & $F_{y}$ & $F_{z}$ & $M_{x}$ & $M_{y}$ & $M_{z}$ \\
\hline Flap + ss + pl & $-70 \%$ & $-83 \%$ & $-79 \%$ & $-92 \%$ & $-82 \%$ & $-26 \%$ \\
Flap + ss & $-71 \%$ & $-84 \%$ & $-77 \%$ & $-91 \%$ & $-82 \%$ & $-30 \%$ \\
Flap + pl & $-58 \%$ & $-80 \%$ & $-62 \%$ & $-74 \%$ & $-50 \%$ & $-17 \%$ \\
Flap & $-75 \%$ & $-85 \%$ & $-59 \%$ & $-78 \%$ & $-54 \%$ & $-23 \%$ \\
\hline
\end{tabular}

it is not present in the lead-lag bending stiffness variation (see Figure 14). This behaviour is probably due to the phase shift between the transfer functions relating hub loads to the different control variables (note that, e.g., the lift variation due to a flap deflection produces an in-quadrature flapping motion, whereas the drag variation is practically in phase with the lead-lag blade deformation).

\section{Conclusion}

The effectiveness of several optimal control strategies aiming at alleviating vibratory loads at helicopter rotor hubs has been examined. These strategies are the result of the combined application of trailing-edge flap and variable-stiffness devices at the blade root and pitch link. Furthermore, the sensitivity of controller performance to the aeroelastic rotor model 


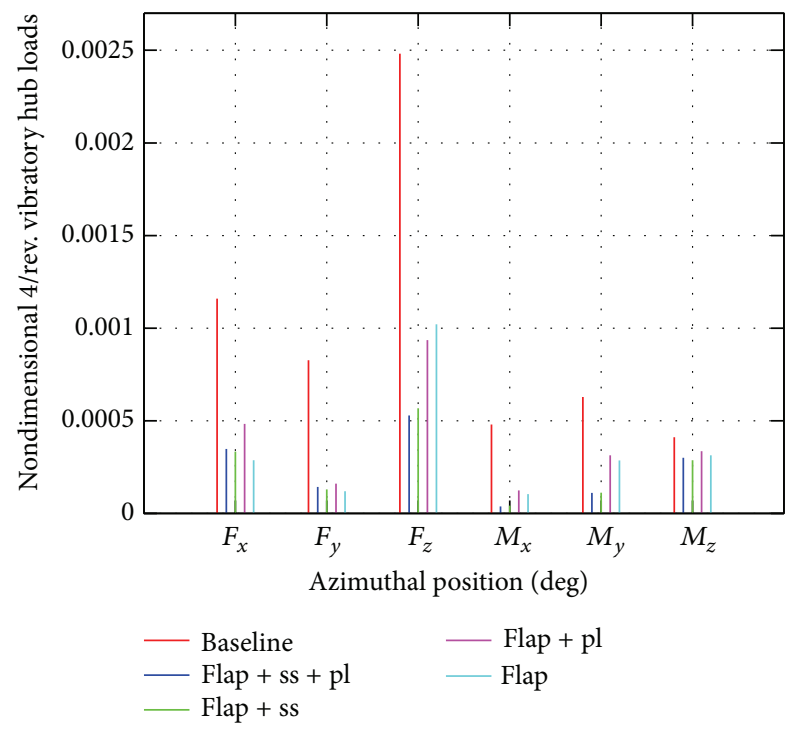

FIGURE 10: Hub loads with feedback, prescribed-wake synthesis, and free-wake inflow validation.

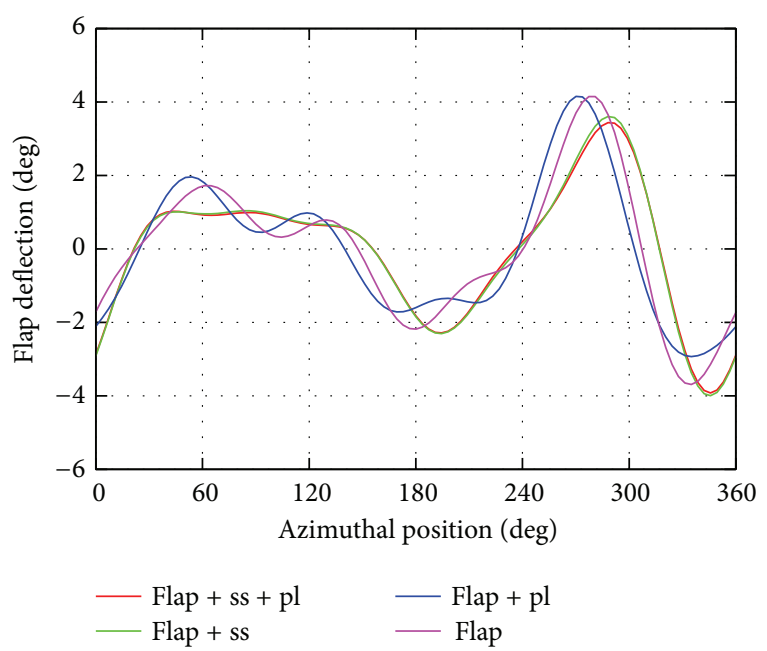

FIgURE 11: Flap deflections time history.

considered in the control-law identification process has been assessed (analysis of robustness with respect to fidelity of the control synthesis rotor aeroelastic model). Satisfactory load reductions have been obtained by the open-loop control actuation applied to the aeroelastic responses predicted by the same rotor model used for control-law identification (specifically, the low-fidelity aeroelastic model considering analytical wake inflow). However, the application of the same cyclic actuation laws to the aeroelastic responses given by a rotor model based on the wake inflow predicted by an aerodynamic free-wake solver has provided unsatisfactory load alleviation. In order to obtain significant vibratory load reductions, the feedback process has been included in the control procedure. In particular, the control matrices obtained through the low-fidelity model have been applied

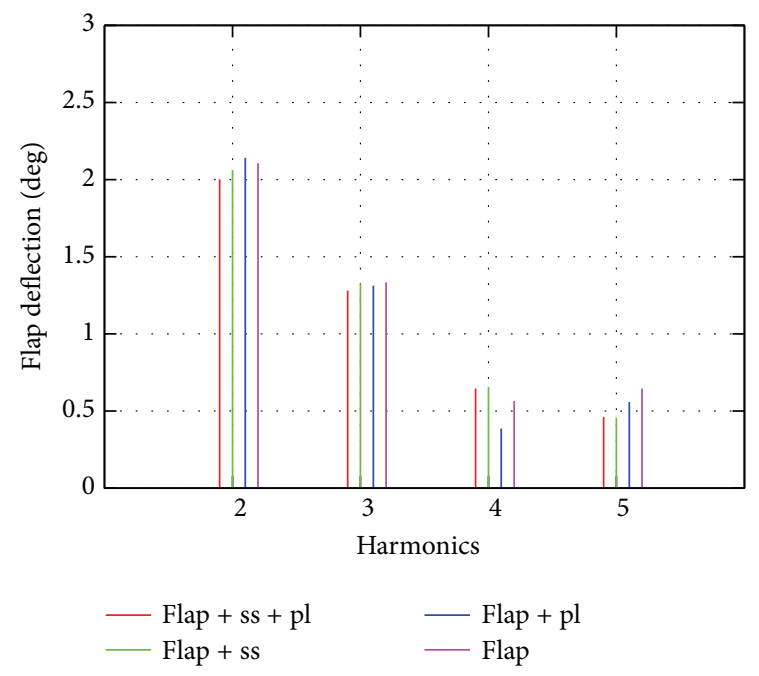

Figure 12: Flap deflections harmonics.

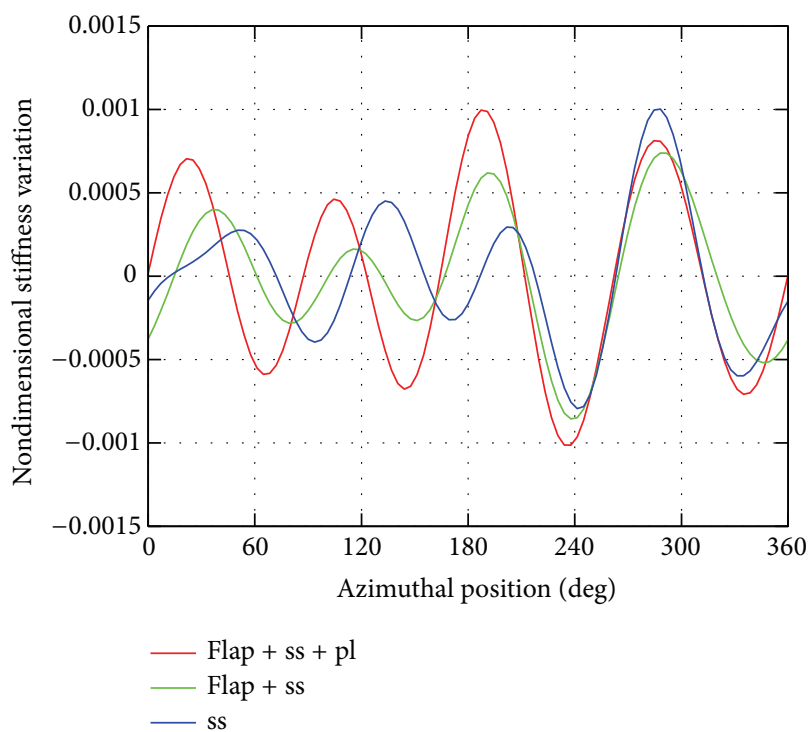

FIGURE 13: Flap smart spring bending stiffness time history.

to update the flap actuation law recursively, exploiting the knowledge of current hub loads (open-loop variable-stiffness actuation laws have been maintained). Better control performances have been obtained enhancing the accuracy of the aeroelastic identification plant, particularly in terms of the contribution from the semiactive actuation of the variablestiffness devices. This analysis has shown that the combined use of flap and blade root variable-stiffness smart spring is the control strategy yielding the highest level of hub loads alleviations, at least for the range of variation of the control variables considered here. Future work is suggested by the observation of the preliminary results obtained by application of the active pitch link: for a better exploitation of the pitch link control potentialities, a dedicated numerical investigation aimed at identifying suitable baseline value 


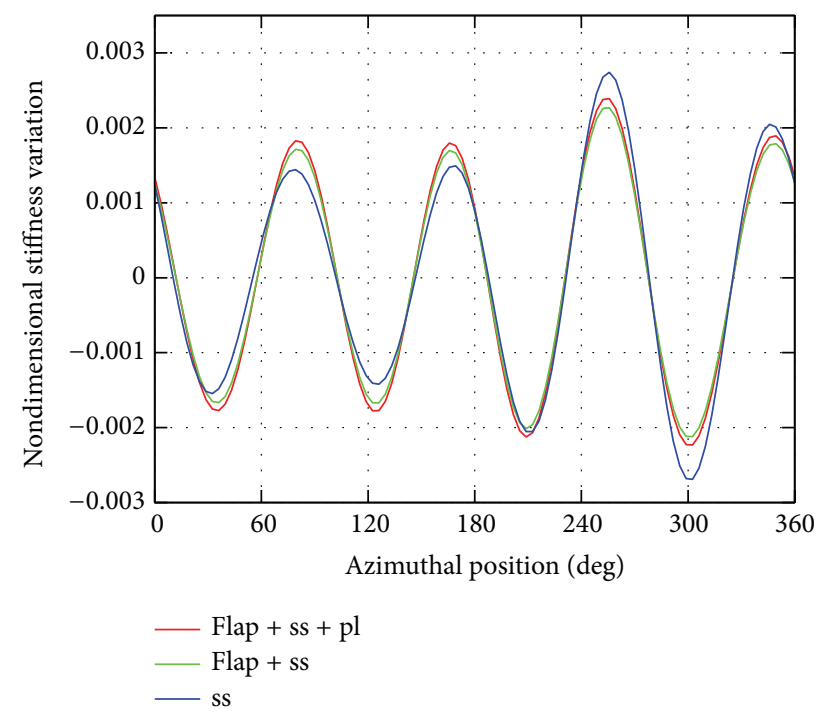

FIGURE 14: Lag smart spring bending stiffness time history.

and range of variation of its stiffness is recommended. To conclude, the combined application of smart devices and trailing-edge flap provides promising results, which would deserve further investigations to confirm their effectiveness and feasibility of actuation.

\section{Nomenclature}

$b$ : $\quad$ Section semichord length

$c: \quad$ Section chord

$\bar{c}: \quad$ Trailing-edge flap chord

$b_{f}: \quad$ Trailing-edge flap span

$\rho: \quad$ Air density

$c_{D}: \quad$ Profile drag coefficient

$C(k): \quad$ Lift deficiency function

D: $\quad$ Section drag

L: $\quad$ Section lift

$L_{n c}: \quad$ Section noncirculatory lift

$L_{c}: \quad$ Section circulatory lift

$T_{j}$ : Theodorsen's aerodynamic coefficients

$u_{T}, u_{P}: \quad$ Blade section velocity components

$e_{A}: \quad$ Dimensionless tensile offset

$\mathbf{f}_{\text {aer }}$ : Dimensionless vector of the generalized aerodynamic forces

$\mathbf{f}_{\mathrm{str}}^{\mathrm{nl}}$ : $\quad$ Dimensionless vector of nonlinear structural forces

$L_{v}, L_{w}: \quad$ Aerodynamic forces per unit length

$M_{\phi}: \quad$ Aerodynamic pitching moment per unit length

$m: \quad$ Blade mass per unit length

M,C,K: Mass, damping, and stiffness matrices

$p_{x}, p_{y}, p_{z}:$ Dimensionless inertial forces per unit length $q_{x}, q_{y}, q_{z}:$ Dimensionless inertial moments per unit length

$J$ : $\quad$ Performance index

q: $\quad$ Vector of the generalized coordinates

$R: \quad$ Rotor radius

T: $\quad$ Dimensionless tension

T: $\quad$ Transfer matrix

u: $\quad$ Vector of control variables

$v, w$ : Dimensionless displacements of the elastic axis

$\mathbf{W}_{u}, \mathbf{W}_{z}$ : Weighting matrices

$x$ : $\quad$ Dimensionless blade span coordinate

z: $\quad$ Vector of hub loads harmonics

$\beta: \quad$ Trailing-edge flap deflection

$\theta: \quad$ Section pitch angle

$\Lambda_{1}: \quad$ Dimensionless flap bending stiffness

$\Lambda_{2}: \quad$ Dimensionless lead-lag bending stiffness

$\kappa: \quad$ Dimensionless torsion rigidity

$\eta: \quad$ Dimensionless mass radii of gyration

$\phi: \quad$ Elastic torsion deflection

$\omega: \quad$ Blade section angular velocity

$\Omega: \quad$ Blade angular velocity.

\section{Conflict of Interests}

The authors declare that there is no conflict of interests regarding the publication of this paper.

\section{References}

[1] P. Anusonti-Inthra and F. Gandhi, "Helicopter vibration reduction through cyclic variations in rotor blade root stiffness," Journal of Intelligent Material Systems and Structures, vol. 11, no. 2, pp. 153-166, 2000.

[2] M. L. Wilbur, P. H. Mirick, W. T. Yeager Jr., C. W. Langston, C. E. S. Cesnik, and S. Shin, "Vibratory loads reduction testing of the NASA/Army/MIT active twist rotor," Journal of the American Helicopter Society, vol. 47, no. 2, pp. 123-133, 2002.

[3] D. Patt, L. Liu, J. Chandrasekar, D. S. Bernstein, and P. P. Friedmann, "Higher-harmonic-control algorithm for helicopter vibration reduction revisited," Journal of Guidance, Control, and Dynamics, vol. 28, no. 5, pp. 918-930, 2005.

[4] U. T. P. Arnold and D. Fürst, "Closed loop IBC results from CH53G flight tests," Aerospace Science and Technology, vol. 9, no. 5, pp. 421-435, 2005.

[5] F. Nitzsche, D. Feszty, D. Waechter et al., "The SHARCS project: smart hybrid active rotor control system for noise and vibration attenuation of helicopter rotor blades," in Proceedings of the 31st European Rotorcraft Forum, pp. 1-52, Firenze, Italy, September 2005.

[6] C. Kessler, "Active rotor control for helicopters: individual blade control and swashplateless rotor designs," CEAS Aeronautical Journal, vol. 1, no. 1-4, pp. 23-54, 2011.

[7] S. R. Viswamurthy and R. Ganguli, "Performance sensitivity of helicopter global and local optimal harmonic vibration controller," Computers \& Mathematics with Applications, vol. 56, no. 10, pp. 2468-2480, 2008.

[8] K. Ravichandran, I. Chopra, B. E. Wake, and B. Hein, “Trailingedge flaps for rotor performance enhancement and vibration 
reduction," Journal of the American Helicopter Society, vol. 58, no. 2, pp. 1-13, 2013.

[9] R. Mallick, R. Ganguli, M. S. Bhat, S. R. Viswamurthy, and R. Ganguli, "Robust design of multiple trailing edge flaps for helicopter vibration reduction: a multi-objective bat algorithm approach," Engineering Optimization, 2014.

[10] M. Gennaretti, M. M. Colella, and G. Bernardini, "Analysis of helicopter vibratory hub loads alleviation by cyclic trailing-edge blade flap actuation," The Aeronautical Journal, vol. 113, no. 1146, pp. 549-556, 2009.

[11] M. Muller, U. T. P. Arnold, and D. Morbitzer, "On the importance and efficiency of $2 / \mathrm{rev}$ IBC for noise, vibration and pitch link load reduction," in Proceedings of the 25th European Rotorcraft Forum, Rome, Italy, September 1999.

[12] M. H. Chia, A. K. Padthe, and P. P. Friedmann, "A parametric study of on-blade control device performance for Helicopter vibration and noise reduction," in Proceedings of the 70th Annual Forum of the American Helicopter Society, Montreal, Canada, May 2014.

[13] F. Nitzsche, A. Grewal, and D. Zimcik, "Structural component having means for actively varying its stiffness to control vibrations," U.S. Patent No. 5,973,440, 999, European Patent EP996570-B1, 2001.

[14] M. Gennaretti, L. Poloni, and F. Nitzsche, "Smart spring' identification for hovering rotor aeroelastic-stability augmentation," The Aeronautical Journal, vol. 107, no. 1071, pp. 233-240, 2003.

[15] D. H. Hodges and E. H. Dowell, "Nonlinear equation for the elastic bending and torsion of twisted nonuniform rotor blades," NASA TN D-7818, 1974.

[16] M. Gennaretti and G. Bernardini, "Novel boundary integral formulation for blade-vortex interaction aerodynamics of helicopter rotors," AIAA Journal, vol. 45, no. 6, pp. 1169-1176, 2007.

[17] G. Bernardini, J. Serafini, M. Molica Colella, and M. Gennaretti, "Analysis of a structural-aerodynamic fully-coupled formulation for aeroelastic response of rotorcraft," Aerospace Science and Technology, vol. 29, no. 1, pp. 175-184, 2013.

[18] T. Theodorsen, "General theory of aerodynamic instability and the mechanism of flutter," NACA Report 496, 1935.

[19] J. M. Greenberg, "Airfoil in sinusoidal motion in a pulsating stream," NACA TN-1326, 1947.

[20] D. H. Hodges and R. A. Ormiston, "Stability of elastic bending and torsion of uniform cantilever rotor blades in hover with variable structural coupling," NASA TN D-8192, 1976.

[21] J. M. Drees, "A theory of airow through rotors and its application to some Helicopter problems," Journal of the Helicopter Association of Great Britain, vol. 3, no. 2, pp. 79-104, 1949.

[22] D. Patt, L. Liu, and P. P. Friedmann, "Rotorcraft vibration reduction and noise prediction using a unified aeroelastic response simulation," Journal of the American Helicopter Society, vol. 50, no. 1, pp. 95-106, 2005.

[23] J. Zhang, Active-passive hybrid optimization of rotor blades with trailing edge flaps [Ph.D. thesis], Department of Aerospace Engineering, Pennsylvania State University, State College, Pa, USA, 2001.

[24] W. Johnson, "Self-tuning regulators for multicyclic control of Helicopter vibration,” NASA TP-1996, 1982.

[25] S. Modini, G. Graziani, G. Bernardini, and M. Gennaretti, "Parallel blade-vortex interaction modelling for helicopter rotor noise control synthesis," International Journal of Aeroacoustics, vol. 13, no. 7-8, pp. 587-606, 2014.
[26] M. Molica Colella, G. Bernardini, and M. Gennaretti, “Tiltrotor wing-root vibratory loads reduction through higher harmonic control actuation," Journal of Aircraft, vol. 49, no. 6, pp. 18131820, 2012.

[27] G. Bernardini, C. Testa, and M. Gennaretti, "Optimal design of tonal noise control inside smart-stiffened cylindrical shells," Journal of Vibration and Control, vol. 18, no. 8, pp. 1233-1246, 2012. 

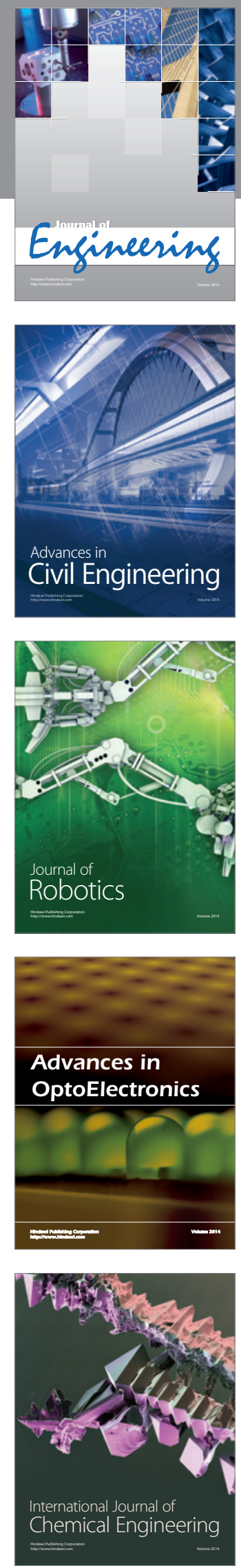

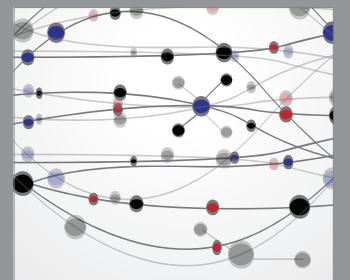

The Scientific World Journal
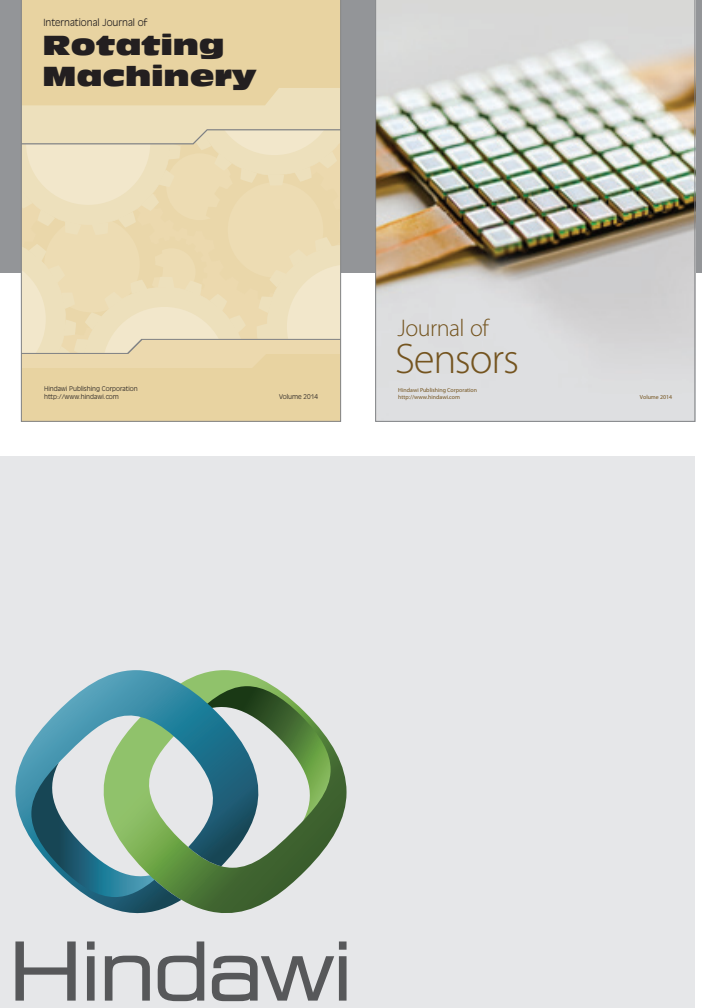

Submit your manuscripts at http://www.hindawi.com
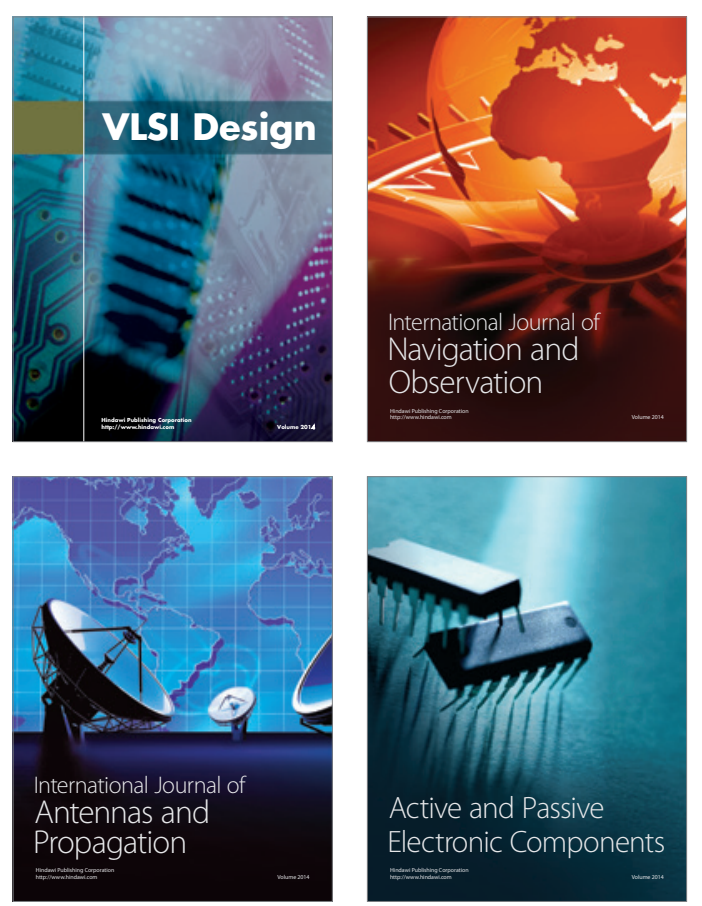
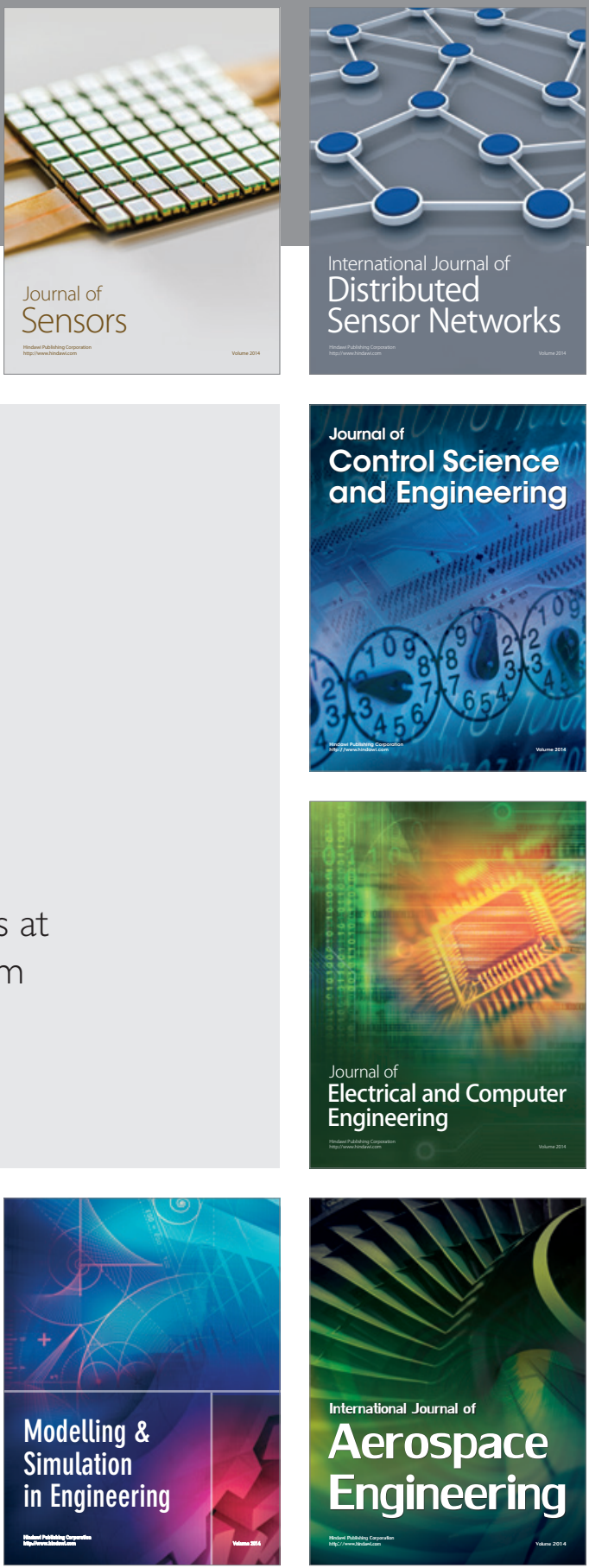

Journal of

Control Science

and Engineering
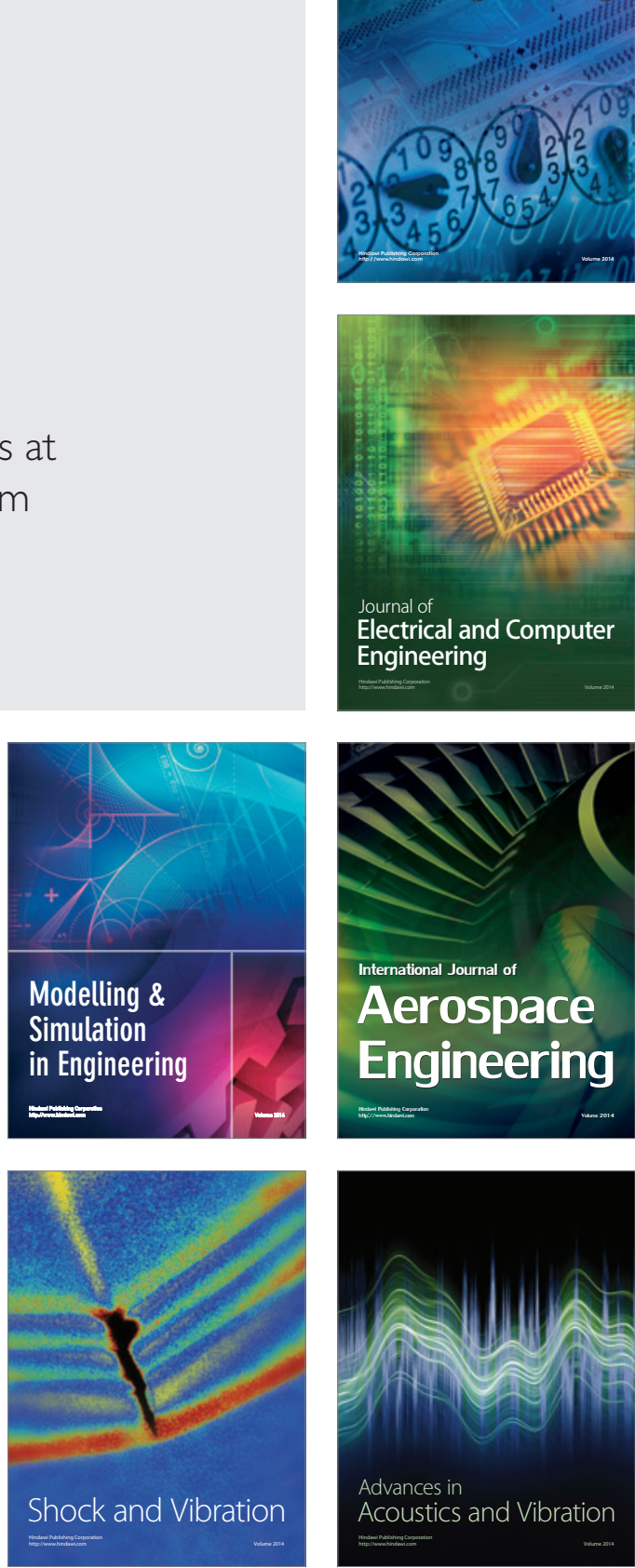